\title{
VALIDACIÓN DEL SCORE MAMÁ Y MACAS EN PACIENTES DE LA REGIÓN AMAZÓNICA DEL ECUADOR.
}

Quezada Galindo José Luis ${ }^{1 *}$, Garay García Louciana Marisol' ${ }^{1}$, Pillco Buestan Silvana Piedad ${ }^{2}$, Peralta Verdugo Jessica Tatiana ${ }^{2}$, Paguay Paredes Diana Cecilia ${ }^{2}$.

DOI: $10.48018 / \mathrm{rmv} . v 31 . i 2.2$

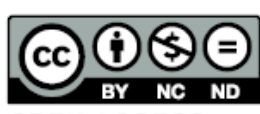
ste artículo está bajo una licencia de Creative Commons de tipo Reconocimien- No comercial - Sin obras derivadas 4.0 International.

OPEN ACCESS

1. Hospital General Macas, Médico tratante del Servicio de Ginecología y Obstetricia. Macas - Ecuador.

2. Hospital General Macas, Médico Residente del Servicio de Ginecología. Macas - Ecuador.

ORCID ID:

Quezada Galindo José Luis orcid.org/0000-0002-1845-8162

Garay García Louciana Marisol orcid.org/0000-0002-9092-0809 Pillco Buestán Silvana Piedad orcid.org/0000-0003-2294-8639 Paguay Paredes Diana Cecilia orcid.org/0000-0002-2044-2207

Peralta Verdugo Jesica Tatiana orcid.org/0000-0002-3858-1299

*Corresponding author: Quezada Galindo José Luis

E-mail: joseluisquezadagalindo@gmail.com

Article history

Received: 5 - May - 2020

Accepted: 14 - Ju1 - 2020

Publish: 1 - Ago - 2020

Recognitions

Winner 1 Place: I Fórum de Investigadores

Hospital Vozandes Quito - HVQ SA.

February 2020

STARD 2015 Check List statement: The author have real the STARD 2015 Check List and the manuscript was prepared and revised according to the STARD 2015 Checklist.

Conflict of interest: All authors declared that there are no conflicts of interest.

Financial disclosure: The authors have no financial relationships relevant to this article to disclose

Authors' contribution: All the authors analyzed and interpreted the data, drafted the article and critically revised the article. All the authors reviewed and approved the final manuscript.

Forma de citar este artículo: Quezada Galindo J, Garay García L, Pillco Buestan S, Peralta Verdugo J, Paguay Paredes D. VALIDACIÓN DEL SCORE MAMÁ Y MACAS EN PACIENTES DE LA REGIÓN AMAZÓNICA DEL ECUADOR. Rev Med Vozandes. 2020; 31 (2): 11 - 17

\section{Resumen}

\section{Introducción}

En el Ecuador, la pre-eclampsia es una de las principales causas de mortalidad materna. Con el objetivo de identificar oportunamente el riesgo en esta población, en el 2017 se desarrolló el score MAMÁ, el cual permite activar claves de emergencia obstétrica y de esta forma proporcionar una atención sistematizada y oportuna. El objetivo de este estudio fue validar los scores MAMÁ y MACAS en pacientes de la región amazónica del Ecuador.

\section{Materiales y métodos}

Se realizó un estudio de cohorte retrospectivo y analítico, fueron elegibles para participar del estudio embarazadas atendidas en el Servicio de Ginecología y Obstetricia del Hospital General de MACAS durante el período: enero 2018 a diciembre 2019. Los scores MAMÁ y MACAS fueron aplicadas a todas las participantes. El análisis estadístico se lo realizo con el software SPSS. Valores de sensibilidad, especificidad, valores predictivos positivos y negativos para cada clave obstétrica fueron calculados. Análisis de regresión logística ajustados para variables confundidoras permitieron identificar el score con mejor desempeño diagnóstico.

\section{Resultados}

Se evaluaron 1259 gestantes con una media de edad de 24,93 años, IMC medio de $27,61 \mathrm{~kg} / \mathrm{m} 2$, los valores medios de las presiones sistólica y diastólica fueron $111,19 \mathrm{mmHg}$ y $70,22 \mathrm{mmHg}$ respectivamente. 101 claves obstétricas fueron activadas, 46 se categorizaron como clave Azul, 14 como clave Amarilla y 41 como clave Roja. Las regresiones logísticas identificaron que un score MAMÁ por sobre 3 puntos aumentaba 18 veces la probabilidad de requerir activación de clave obstétrica, mientas que, con el score MACAS la misma puntuación aumentó en 20 la probabilidad de activar las claves obstétricas.

\section{Conclusiones}

Ambos escores son adecuados para el reconocimiento de gestantes con riesgo obstétrico, el escore MACAS presentó una mejor especificidad, respecto a las claves obstétricas. Estos resultados deberán ser validados en cohortes mayores y de forma prospectiva con el objetivo de establecer el mejor escore en subgrupos de pacientes.

Palabras clave: Riesgo obstétrico, Embarazo, Ácido Láctico, Pre-eclampsia. 
Keywords: Obstetric risk, Pregnancy, Lactic acid, Pre-eclampsia.

\section{Abstract}

\section{VALIDATION OF THE MAMÁ AND MACAS SCORE IN PATIENTS FROM THE AMAZON REGION OF ECUADOR.}

\begin{abstract}
Introduction
In Ecuador, preeclampsia is one of the leading causes of maternal mortality. In order to identify the risk in this population in a timely manner, in 2017 the MAMÁ Score was developed, which allows to activate obstetric emergency keys and thus provide a systematized and timely care. The objective of this study was to validate the MAMÁ and MACAS score in patients in the Amazon region of Ecuador.
\end{abstract}

\begin{abstract}
Materials and methods
A retrospective and analytical cohort study was conducted, they were eligible to participate in the pregnant study attended by the Ginecology and Obstetrics Service of the MacAS General Hospital in the period: January 2018 to December 2019. The MAMÁ and MACAS scores were applied to all participants. Statistical analysis was performed with SPSS software. Sensitivity values, specificity, positive and negative predictive values for each obstetric key were calculated. Adjusted logistic regression analysis for confusing variables made it possible to identify the score with better diagnostic performance.
\end{abstract}

\begin{abstract}
Results
1259 pregnant women were evaluated an average age of 24.93 years, average BMM of $27.61 \mathrm{Kg} / \mathrm{m} 2$, mean systolic and diastolic pressure values were $111.19 \mathrm{mmHg}$ and $70.22 \mathrm{mmHg}$ respectively. 101 obstetric keys were activated, where 46 were categorized as Blue Key, 14 as Yellow Key, and 41 Red Key. Logistic regressions identified that an score MAMÁ by abou 3 points increased by 18 times the probability of requiring obstetric key activation, while with the MACAS score the same score increased by 20 times the probability of activating the obstetric keys.
\end{abstract}

\section{Conclusions}

Both scores are suitable for the recognition of pregnant women with obstetric risk, the MACAS score presented a better specificity, with respect to obstetric keys. These results should be validated in larger cohorts and prospectively with the aim of establishing the best score in patient subgroups.

\section{Introducción}

La gestación es un proceso complejo, en el que se llevan a cabo cambios importantes en la mujer, tanto a nivel fisiológico como psicológico. Los eventos biológicos, van desde cambios en el aspecto físico de la mujer (progresivo aumento de peso y de volumen), hasta cambios fisiológicos en los sistemas: cardiovascular, urinario, digestivo, respiratorio, de coagulación y órganos reproductivos (1).

Se ha determinado que el promedio de peso ganado durante el embarazo es de 12,5 kg; este nivel de ganancia está asociado con resultados favorables en mujeres saludables. El corazón y la circulación presentan adaptaciones fisiológicas importantes desde las primeras semanas del embarazo. El gasto cardiaco se incrementa hasta en un $50 \%$ en comparación con la mujer no gestante, atribuyéndose estas modificaciones a una elevación de la frecuencia cardiaca (15 a $25 \%$ mayor que en la mujer no embarazada), al volumen latido, que se encuentra elevado en 25 a $30 \%$ al final del embarazo, y finalmente a una disminución de la resistencia vascular periférica, en $20 \%$, aproximadamente ${ }^{(2)}$.

A nivel renal se produce dilatación de la pelvis renal, cálices y uréteres, provocando aumento del espacio muerto urinario. El incremento del espacio muerto urinario unido al crecimiento de la vascularización renal y al mayor volumen intersticial ocasionan aumento en la longitud del riñón, de aproximadamente 1 a $1.5 \mathrm{~cm}$, en comparación con el riñón de la mujer no gestante ${ }^{(3)}$. 
El estómago se modifica debido a factores mecánicos y hormonales; el elemento mecánico es el útero ocupado y el factor hormonal, la progesterona, que disminuye el peristaltismo gástrico e intestinal; como resultado del factor hormonal, se producirá retraso en el vaciamiento gástrico y, en el intestino, una mayor absorción, debido a la lentitud en el tránsito intestinal (1).

El hígado no experimenta modificaciones morfológicas pero el flujo sanguíneo de la vena porta aumenta significativamente a partir de las 28 semanas de gestación, aunque el flujo de la arteria hepática no se altera; es evidente una reducción en la actividad de CYP1A2. Este efecto sería por acción de la progesterona, la cual juega un rol importante en la regulación del metabolismo ${ }^{(4)}$.

Por resonancia magnética se ha demostrado que el riego sanguíneo cerebral bilateral en las arterias cerebrales media y posterior disminuye progresivamente hasta el tercer trimestre: se desconoce el mecanismo e importancia clínica de este hallazgo, aunque podría explicar la disminución de la memoria durante el embarazo.

A partir de la octava semana ocurren modificaciones en las capacidades, volúmenes y ventilaciones pulmonares, por efecto hormonal y modificaciones mecánicas y anatómicas ${ }^{(5)}$.

En la piel, la influencia de las hormonas del embarazo producen: prurito, cloasma o melasma y estrías. Las hormonas sexuales aumentan su producción y la mujer muestra un desbalance inmunológico, lo que la hace susceptible a agresiones con relación a la mujer no grávida. En cuanto a los cambios psicológicos, éstos son más importantes a medida que el embarazo progresa, al igual que en el parto y el puerperio (6).

En Ecuador, la primera causa de mortalidad materna gira en torno a las enfermedades hipertensivas y la pre-eclampsia, seguida de las hemorragias e infecciones. En el país, se estima que 30 mujeres por cada 100 mil habitantes al año presentan estas complicaciones. La mayoría son prevenibles o tratables, algunas pueden estar presentes desde antes del embarazo pero se agravan con la gestación (7).

En el Ecuador en el año 2017 se desarrolló el score MAMÁ con el objetivo de identificar y notificar oportunamente claves de riesgo obstétrico, de esta forma la atención en pacientes obstétricas se sistematiza. ${ }^{\left({ }^{8}\right)}$.

En el Hospital General de Macas se atiende pacientes de diferentes poblaciones en su mayoría de la etnia Shuar, en la práctica diaria se ha podido observar que muchas pacientes sanas presentan valores por encima del rango normal en el score MAMÁ sobre todo a expensas de la tensión arterial materna, por lo que se planteó la posibilidad de que en la población indígena exista una tensión arterial media inferior a la de la población mestiza, por tal motivo se propuso desarrollar un nuevo score con parámetros que se ajustan a nuestra población, al cual se denominó score MACAS incluyendo al lactato sérico como otro parámetro ya que ha demostrado ser útil como un predictor de gravedad de las pacientes con complicaciones obstétricas. El objetivo de este estudio fue validar los dos scores y la activación de claves obstétricas en pacientes de la región amazónica.

\section{Materiales y métodos}

\section{Selección de la cohorte}

Este fue un estudio de cohorte retrospectiva y de tipo analítico. Fueron elegibles pacientes obstétricas, atendidas en el Servicio de Ginecología y Obstetricia del Hospital General de MACAS entre enero de 2018 y diciembre del 2019. Pacientes en las que no se confirmó el embarazo, de etnia diferente a la Shuar o mestiza y con prontuarios médicos incompletos fueron excluidas.

\section{Validación score MAMÁ y MACAS}

El score MAMÁ consta de los siguientes parámetros: Frecuencia cardíaca, tensión arterial, frecuencia respiratoria, temperatura, saturación de Oxígeno, estado de conciencia y proteinuria; mientras que el score MACAS además de los parámetros citados se suma la determinación de lactato sérico con modificaciones en los rangos de tensión arterial. En ambos casos, las pacientes con puntaje de 3 son consideradas como alto riesgo y deben ser abordadas de manera más detenida para valorar la activación de una clave obstétrica. En un trabajo anterior se determinó que el score MACAS tuvo una sensibilidad similar, pero mejor especificidad para detectar trastornos hipertensivos del embarazo (THE: pre-eclampsia severa y eclampsia) comparado con el score MAMÁ en pacientes de etnia Shuar y Mestizas. Se observó que un score MACAS por encima de 3 puntos, se asoció con 34,45 veces más probabilidad de presentar un THE (OR=34,45; IC95\%: 13,56-108,99); por otra parte, se evidenció que la presencia del antecedente familiar de pre-eclampsia aumenta 15 veces la probabilidad de tener un THE, razón por la cual en el presente estudio se tomará el punto de corte de 3 para determinar un valor predictor para la activación de las claves obstétricas ${ }^{(9)}$.

\section{Análisis Estadístico}

Los datos fueron analizados en el programa SPSS versión 20, las variables cualitativas se expresaron como frecuencias absolutas y relativas utilizándose la prueba chi cuadrado para evaluar asociación entre las mismas; mientas que las variables cuantitativas se expresaron en medias \pm desviación estándar, evaluando diferencia estadística a través de la prueba t-Student. Se realizaron 2 análisis de regresión logística para activación de claves obstétricas el primero ajustado por: edad, etnia, peso, talla, índice de masa corporal, antecedente familiar de preeclampsia y score MAMÁ. Mientras que el segundo tuvo como covariables: edad, etnia, peso, talla, índice de masa corporal, antecedente familiar de preeclampsia y score MACAS.

\section{Aspectos éticos}

Este estudio sigue las directrices STARD para la publicación de estudios relativos a la precisión diagnóstica y fue aprobado por el comité de ética del Hospital General de Macas. 


\section{Resultados}

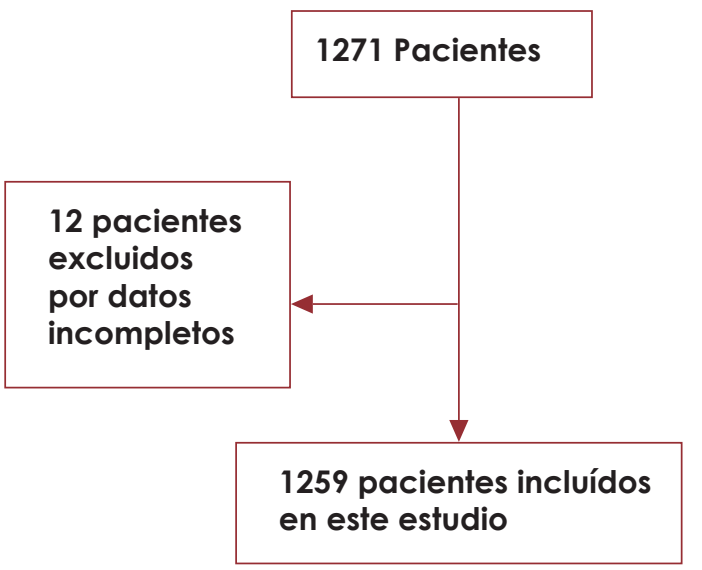

Figura 1. Flow chart de pacientes elegibles y que fueron incluidas en el estudio

(enero 2018 - diciembre 2019)

Fuente: elaborado por los autores

\section{Caracterización de la muestra}

Tabla 1. Comportamiento de las variables en estudio.

$\begin{array}{lcc}\text { Características } & \text { Media } & \begin{array}{c}\text { Desviación } \\ \text { típica }\end{array} \\ \text { Edad (años) } & 24,93 & 7,59 \\ \text { Peso (Kg) } & 62,86 & 10,56 \\ \text { Talla (metros) } & 1,51 & , 06 \\ \text { IMC (Kg/m2) } & 27,71 & 4,34 \\ \text { PAS (mmHg) } & 111,19 & 14,17 \\ \text { PAD (mmHg) } & 70,22 & 10,90 \\ \text { PAM (mmHg) } & 83,88 & 11,06 \\ \text { Score MAMA } & , 89 & 1,84 \\ \text { Score MACAS } & , 85 & 1,73\end{array}$

IMC: Índice de masa corporal; PAS: Presión arterial sistólica; Presión arterial diastólica; Presión arterial media.

Fuente: elaborado por los autores

Entre 1 de enero de 2018 y 31 de diciembre de 2019, se atendieron, 1271 pacientes obstétricas en el Servicio de Ginecología y Obstetricia del Hospital General de Macas, de las cuales $12(0,94 \%)$ fueron excluidas por datos incompletos en los prontuarios. Por tanto, nuestra muestra se compone de 1259 pacientes obstétricas.

Al no encontrarse diferencias entre las etnias estudiadas en las variables del score MAMÁ y score MACAS se decidió realizar un análisis único de las variables, los datos demográficos y características de las pacientes se expresan en la tabla 1. La media de edad fue de 24,93 años, del mismo modo la media del IMC fue $27,61 \mathrm{~kg} / \mathrm{m}^{2}$, mientras que la media de la presión sistólica y diastólica fueron: $111,19 \mathrm{mmHg}$ y $70,22 \mathrm{mmHg}$.
Tabla 2. Antecedentes personales y familiares de las gestantes en estudio.

\begin{tabular}{lcc}
\multicolumn{1}{c}{ Característica } & Recuento & $\%$ \\
$\begin{array}{l}\text { Antecedente personal } \\
\text { de Preeclampsia }\end{array}$ & 7 & $0,6 \%$ \\
$\begin{array}{l}\text { Antecedente familiar de } \\
\text { Preeclampsia }\end{array}$ & 9 & $0,7 \%$ \\
$\begin{array}{l}\text { Antecedente de Emba- } \\
\text { razo Múltiple }\end{array}$ & 1 & $0,1 \%$ \\
$\begin{array}{l}\text { Antecedente de Hiper- } \\
\text { tensión arterial }\end{array}$ & 1 & $0,1 \%$ \\
$\begin{array}{l}\text { Antecedente de Diabe- } \\
\text { tes Mellitus } \\
\text { Ingesta de Calcio }\end{array}$ & 1256 & $99,8 \%$ \\
$\begin{array}{l}\text { Ingesta de Ácido Acetil- } \\
\text { silícico }\end{array}$ & 2031 & $\mathbf{7 3 , 9 \%}$ \\
Total & $\mathbf{1 2 5 9}$ & $\mathbf{1 0 0 , 0 \%}$ \\
\hline
\end{tabular}

Tabla 3. Claves obstétricas activadas en las gestantes en estudio.

\begin{tabular}{lcc}
\multicolumn{1}{c}{ Clave Obstétrica } & Recuento & $\%$ \\
Azul & 46 & $3,7 \%$ \\
Amarilla & 14 & $1,1 \%$ \\
Roja & 41 & $3,3 \%$ \\
Total & 1259 & $100,0 \%$ \\
\hline \multicolumn{2}{c}{ Fuente: elaborado por los autores }
\end{tabular}

Los antecedentes personales y familiares de las gestantes se muestran en la tabla 2, el 0,6\% tuvo antecedente personal de preeclampsia y el $0,7 \%$ antecedente familiar, el $0,1 \%$ indicó tener el antecedente de embarazo múltiple, $0,1 \%$ de hipertensión arterial, $0,2 \%$ de diabetes mellitus, el $73,9 \%$ refirió la ingesta de calcio y el $16,1 \%$ de ácido acetilsilícico

Se activaron 101 claves obstétricas, de las cuales 46 fueron clasificadas como Clave Azul (Preeclampsia con criterios de severidad/Eclampsia), 14 casos presentaron Clave amarilla (Sepsis) y 41 Clave Roja (Hemorragia Post parto) (Tabla 3).

\section{Score MAMÁ Y MACAS}

En la tabla 4 se demuestran los valores de sensibilidad (S), especificidad (E), valor predictivo positivo (VPP) y valor predictivo negativo (VPN) para la activación de clave azul según el score MAMÁ: S: $63 \%, E: 85 \%$, VPP: $14 \%$ y VPN: $98 \%$ respectivamente y para el score MACAS: S: $65 \%$, E: $87 \%$, VPP: $16 \%$ y VPN: $99 \%$, de igual manera, en la tabla 5 y 6 se especifican los valores obtenidos para la activación de la clave amarilla y roja para el score MAMÁ y para el score MACAS respectivamente. 
Tabla 4. Sensibilidad y especificidad de los scores MAMÁ y MACAS para determinar clave obstétrica azul Clave Azul

\begin{tabular}{|c|c|c|c|c|c|c|c|}
\hline \multirow[t]{2}{*}{ Score } & Puntaje & Si & No & & & & \\
\hline & & n. (\%) & n. (\%) & S (\%) & $E(\%)$ & VPP (\%) & VPN (\%) \\
\hline \multirow[t]{2}{*}{ MAMÁ } & $\geq 3$ & $29(63,0)$ & $117(14,6)$ & 63 & 85 & 14 & 98 \\
\hline & $<3$ & $17(37)$ & $1036(85,4)$ & - & - & - & - \\
\hline \multirow[t]{2}{*}{ MACAS } & $\geq 3$ & $30(65,2)$ & $162(13,4)$ & 65 & 87 & 16 & 99 \\
\hline & $<3$ & $16(34,8)$ & $1051(86,6)$ & - & - & - & - \\
\hline
\end{tabular}

Fuente: elaborado por los autores

Tabla 5. Sensibilidad y especificidad de los scores MAMÁ y MACAS para determinar clave obstétrica amarilla Clave Amarilla

$\begin{array}{cccccccc}\text { Score } & \text { Puntaje } & \text { Si } & \text { No } & & & \\ & & \text { n. (\%) } & \text { n. (\%) } & \text { S (\%) } & \text { E (\%) } & \text { VPP (\%) } & \begin{array}{c}\text { VPN } \\ \text { (\%) }\end{array} \\ \text { MAMÁ } & \geq 3 & 11(78,6) & 195(15,7) & 79 & 84 & 5 & 100 \\ & <3 & 3(21,4) & 1050(84,3) & - & - & - & - \\ \text { MACAS } & \geq 3 & 11(78,6) & 181(14,5) & 65 & 87 & 16 & 99 \\ & <3 & 3(21,4) & 1064(85,5) & - & - & - & - \\ \text { S: Sensibilidad, E: especificidad, VPP: valor predictivo positivo, VPN: Valor predictivo negativo } & & \end{array}$

Fuente: elaborado por los autores

Tabla 6. Sensibilidad y especificidad de los scores MAMÁ y MACAS para determinar clave obstétrica roja en las gestantes en estudio.

\begin{tabular}{|c|c|c|c|c|c|c|c|}
\hline \multicolumn{8}{|c|}{ Clave Roja } \\
\hline \multirow[t]{2}{*}{ Score } & Puntaje & Si & No & & & & \\
\hline & & n. (\%) & n. (\%) & S (\%) & $E(\%)$ & VPP (\%) & VPN (\%) \\
\hline \multirow[t]{2}{*}{ MAMÁ } & $\geq 3$ & $25(61,0)$ & $181(15,0)$ & 61 & 85 & 12 & 98 \\
\hline & $<3$ & $16(39,0)$ & $1037(85,1)$ & - & - & - & - \\
\hline \multirow[t]{2}{*}{ MACAS } & $\geq 3$ & $23(56,1)$ & $169(13,9)$ & 56 & 86 & 12 & 98 \\
\hline & $<3$ & $18(43,9)$ & $1049(86,1)$ & - & - & - & - \\
\hline
\end{tabular}

Fuente: elaborado por los autores

Tabla 7. Regresión logística para determinar la activación de claves obstétricas en las gestantes en estudio.

Odds Ratio

Score

MAMA

Puntaje

$$
\begin{aligned}
& <3 \text { (Bajo) } \\
& \geq 3 \text { (Alto) }
\end{aligned}
$$

MACAS

$$
\begin{aligned}
& <3 \text { (Bajo) } \\
& \geq 3 \text { (Alto) }
\end{aligned}
$$

\begin{abstract}
IC $95 \% *$
\end{abstract}
1,00

$18,38(11,23-30,08)$
Odds Ratio

Valor $\mathrm{p} \quad$ IC $95 \% * *$

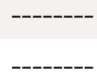

1,00

$$
20,09(12,23-33,00)
$$$$
<0,001
$$

* Modelo 1: Ajustado por edad, etnia, peso, talla, índice de masa corporal, antecedente familiar de preeclampsia y Score MAMÁ.

** Modelo 2: Ajustado por edad, etnia, peso, talla, índice de masa corporal, antecedente familiar de preeclampsia y Score MACAS. 
SCORE MAMÁ

\begin{tabular}{|c|c|c|c|c|c|c|c|c|c|}
\hline \multirow{2}{*}{ Parámetros } & \multicolumn{7}{|c|}{ Puntaje } & \multirow{2}{*}{ Parámetro } & \multirow{2}{*}{$\begin{array}{l}\text { Puntaje } \\
\text { parcial }\end{array}$} \\
\hline & 3 & 2 & 1 & 0 & 1 & 2 & 3 & & \\
\hline $\mathrm{FC}$ & $\leq 50$ & & $51-59$ & $60-100$ & $101-110$ & $111-119$ & $\geq 120$ & $\mathrm{FC}$ & \\
\hline Sistólica & $\leq 70$ & $71-89$ & & $90-139$ & & $140-159$ & $\geq 160$ & Sistólica & \\
\hline Diastólica & $\leq 50$ & $51-59$ & & $60-85$ & $86-89$ & $90-109$ & $\geq 110$ & Diastólica & \\
\hline $\mathrm{FR}$ & $\leq 11$ & & & 12. -22 & & $23-29$ & $\geq 30$ & $\mathrm{FR}$ & \\
\hline TOC C & & $\leq 35,5$ & & $35,6-37,5$ & $37,6-38,4$ & & $\geq 38,5$ & TOC & \\
\hline Sat 02 & $\leq 85$ & $86-89$ & $90-93$ & $94-100$ & & & & Sat 02 & \\
\hline $\begin{array}{l}\text { Estado de } \\
\text { Conciencia }\end{array}$ & & $\begin{array}{c}\text { confusa/ } \\
\text { agitada }\end{array}$ & & Alerta & $\begin{array}{c}\text { responde a la } \\
\text { voz/somnolienta }\end{array}$ & $\begin{array}{c}\text { responde al } \\
\text { dolor/estupo } \\
\text { rosa }\end{array}$ & $\begin{array}{c}\text { no } \\
\text { responde }\end{array}$ & $\begin{array}{l}\text { Estado de } \\
\text { Conciencia }\end{array}$ & \\
\hline Proteinuria & & & & Negativo & Positivo & & & Proteinuria + & \\
\hline & & & & & & & & & \\
\hline
\end{tabular}

Anexo 1. Score MAMÁ

SCORE MACAS

\begin{tabular}{|c|c|c|c|c|c|c|c|c|c|}
\hline \multirow{2}{*}{ Parámetros } & \multicolumn{7}{|c|}{ Puntaje } & \multirow{2}{*}{ Parámetro } & \multirow{2}{*}{$\begin{array}{l}\text { Puntaje } \\
\text { parcial }\end{array}$} \\
\hline & 3 & 2 & 1 & 0 & 1 & 2 & 3 & & \\
\hline $\mathrm{FC}$ & $\leq 50$ & & $51-59$ & $60-100$ & $101-110$ & $111-119$ & $\geq 120$ & $\mathrm{FC}$ & \\
\hline Sistólica & $\leq 69$ & & $70-89$ & $90-139$ & & $140-159$ & $\geq 160$ & Sistólica & \\
\hline Diastólica & $\leq 49$ & & $50 \cdot 59$ & $60-80$ & $81-89$ & $90 \cdot 109$ & $\geq 110$ & Diastólica & \\
\hline $\mathrm{FR}$ & $\leq 11$ & & & 12. -22 & & $23 \cdot 29$ & $\geq 30$ & $F R$ & \\
\hline $\mathrm{T} \cong \mathrm{C}$ & & $\leq 35,5$ & & $35,6-37,5$ & $37,6-38,4$ & & $\geq 38,5$ & $\mathrm{~T} \cong \mathrm{C}$ & \\
\hline Sat 02 & $\leq 85$ & $86-89$ & $90-93$ & $94-100$ & & & & Sat 02 & \\
\hline $\begin{array}{l}\text { Estado de } \\
\text { Conciencia }\end{array}$ & & $\begin{array}{c}\text { confusa/ } \\
\text { agitada }\end{array}$ & & Alerta & $\begin{array}{c}\text { responde a la } \\
\text { voz/somnolienta }\end{array}$ & $\begin{array}{c}\text { responde al } \\
\text { dolor/estupo } \\
\text { rosa }\end{array}$ & $\begin{array}{l}\text { no } \\
\text { responde }\end{array}$ & $\begin{array}{c}\text { Estado de } \\
\text { Conciencia }\end{array}$ & \\
\hline Proteinuria & & & & Negativo & Positivo & & & Proteinuria + & \\
\hline Lactato $\mathrm{mMol} / \mathrm{L}$ & & & & $<1$ & 1. $-1,9$ & $2-3,9$ & $\geq 4$ & & \\
\hline
\end{tabular}

Anexo 2. Score MACAS

Se realizaron dos modelos de regresión logística para determinar la activación de las claves obstétricas, donde se pudo observar que un score MAMÁ por sobre 3 puntos aumenta la probabilidad en 18 veces de ameritar la activación de una de las claves obstétricas (OR= 18,38; IC95\%: 11,23-30,08), mientras que un score MACAS por encima de los 3 puntos tiene 20 veces más riesgo activar las claves obstétricas (OR=20,09; IC95\%: 12,23-33,08) (Tabla 7).

\section{Discusión}

En Ecuador la morbimortalidad materna ha sido un problema de salud pública que ha sido abordado desde diferentes perspectivas con la finalidad de reducir el impacto que tiene sobre las gestantes y el producto de la gestación (7), razón por la cual se han propuesto diferentes estrategias con este fin, una de estas ha sido el score MAMÁ el cual es una escala que permite clasificar a las mujeres embarazadas según el riesgo obstétrico que presenten, de igual manera, se reconoce que existen regiones en el país que socio-demográficamente son diferentes, motivo por el cual se desarrolló el score MACAS para las gestantes de la región amazónica ${ }^{(9)}$.

Al evaluar la S, E, VPP y VPN de los scores MAMÁ y MACAS con un valor mayor a 3 obtuvimos un desempeño aceptable, con sensibilidades que superaron en la mayoría de los análisis el 60\%, E mayor al 70\%, VPP superior al $5 \%$ y un VPN superior al $90 \%$.

Del mismo modo, los modelos de regresión permitieron establecer una asociación entre un puntaje mayor a 3 en los scores MAMÁ y MACAS con la probabilidad de activar claves obstétricas, 18 veces y 20 veces respectivamente, este último ligeramente superior, por lo cual se recalca la utilidad de este score en esta población específica, este comportamiento también se evidenció en un estudio previo en donde se evaluó el valor predictivo que tenían estos scores en pacientes con trastornos hipertensivos, en donde el score MACAS fue ligeramente superior que el MAMÁ (9).

En Ecuador, otro estudio ha demostrado la utilidad del score MAMÁ en las mujeres de la ciudad de Salcedo con una E del $97 \%$, S: $100 \%$, VPP: $86 \%$ y VPN del $100 \%{ }^{(10)}$.

Del mismo modo, un reporte realizado en la 
ciudad de Guayaquil evaluó la utilidad del score MAMÁ para la detección de riesgo de morbilidad obstétrica, con los siguientes parámetros: S del 70\%, E: 94\%, VPP: $86 \%$ y un VPN del $85 \%$, concluyendo con estos datos que es una herramienta útil y que debe utilizarse de forma rutinaria en los hospitales del país, tal como se documentó en el presente estudio (11).

Estudio realizado en la ciudad de Macará se identificó que las complicaciones obstétricas se redujeron de 45,12 a $25,74 \%$ con la aplicación del score MAMA, remarcando la importancia de diagnósticos adecuados y oportunos ${ }^{(12)}$.

\section{Conclusión}

Se observó que tanto el score MAMÁ y el score MACAS son útiles como screening para detectar pacientes con riesgo obstétrico (Hemorragia, trastornos hipertensivos y sepsis) resaltando que el score MACAS tiene una mejor especificidad. En cuanto a las claves obstétricas activadas la primera causa de morbilidad materna son los trastornos hipertensivos, seguidos de la hemorragia post parto y en número menor de casos la sepsis, coincidiendo con los datos a nivel nacional. Por lo tanto, el score MACAS se convierte en una herramienta útil e innovadora que podría optimizar el abordaje del cuidado materno-fetal en poblaciones de riesgo, sobre todo al implementar la determinación de lactato sérico para complementar la evaluación metabólica.

\section{Referencias}

1. Soma-Pillay P, Catherine N-P, Tolppanen H, 5. Bhatia P, Chhabra S. Physiological and anaMebazaa A, Tolppanen H, Mebazaa A. Phy siological changes in pregnancy. CardiovasC 2020];27(2):89-94. Disponible en: https://www. ncbi.nlm.nih.gov/pmc/articles/PMC4928162/

2. Lecorguillé $M$, Jacota $M$, Lauzon-Guillain B de, Forhan $A$, Cheminat $M$, Charles $M-A$ et al. An association between maternal weight change in the year before pregnancy and infant birth weight: ELFE, a French national birth cohort study. PLOS Med [Internet]. 20 de agosto de 2019 [citado 3 de agosto de 2020];16(8):e1002871. Disponible en: https://journals.plos.org/plosmedicine/ article?id=10.1371/journal.pmed.1002871

3. Cheung KL, Lafayette RA. Renal Physiology of Pregnancy. Adv Chronic Kidney Dis [In ternet]. mayo de 2013 [citado 3 de agos to de 2020];20(3):209-14. Disponible en: https://www.ncbi.nlm.nih.gov/pmc/articles/ PMC4089195/

4. Westbrook RH, Dusheiko G, Williamson C. Pregnancy and liver disease. I Hepatol [Internet]. abril de 2016 [citado 3 de agosto de 2020];64(4):933-45. Disponible en: https://linkinghub.elsevier.com/retrieve/pii/ S0168827815007886 tomical changes of pregnancy: Implications for anaesthesia. Indian J Anaesth. 1 de septiembre de 2018;62:651.

6. Tyler K. Physiological Skin Changes During Pregnancy. Clin Obstet Gynecol. 16 de diciembre de 2014;58.

7. MSP. Estimación de la Razón de Mortalidad Materna en el Ecuador [Internet]. 2017. Disponible en: https://www.ecuadorencifras.gob. ec/documentos/web-inec/Poblacion_y_Demografia/Nacimientos_Defunciones/2016/ RMM_Nota_metodologica_INEC_2016.pdf

8. ALTAMIRANO C. "Impacto del score mama sobre la mortalidad materna en mujeres embarazadas con más de 20 semanas de embarazadas con más de 20 semanas de
gestación en el servicio de emergencia del gestación en el servicio de emergencia del
hospital yerovi mackuart de la ciudad de hospital yerovi mackuart de la ciudad de de 2018]. Disponible en: http://dspace.uniandes.edu.ec/bitstream/123456789/5769/1/ PIUAMED021-2017.pdf

9. Quezada J, Louciana G, Molina A, Auquilla $R$, Cárdenas D, Montero $M$, et al. Trastornos hipertensivos y utilidad del score MAMÁ y score MACAS en gestantes mestizas y de etnia SHUAR. Arch Venez Farmacol Ter. 2019;38(3):1-8.
10. López A Augusta C. Impacto del score mama sobre la mortalidad materna en mujeres embarazadas con más de 20 semanas de gestación en el servicio de emergencia del Hospital Yerovi Mackuart en la ciudad de Salcedo [Internet] [Tesis de grado]. [AmbatoEcuador]: universidad regional autónoma de los andes: 2017 [citado 3 de agosto de 2020]. Disponible 201 : http://localhost:8080/xmlui/ handle/123456789/5769

11. Cruz, D, Macías D. Utilidad del Score MAMÁ como herramienta para detección de riesgo de morbilidad obstétrica en pacientes embarazadas que acuden al servicio de Emergencia del Hospital «Teodoro Maldonado Carbo» de Diciembre del 2016 a Abril del 2017 [Tesis de pregrado]. [GuayaquilEcuador]: Universidad católica de Santiago de Guayaquil; 2017.

12. Navarro, L. Estudio comparativo de complicaciones obstétricas en embarazadas atendidas con la aplicación del score mamá y sin él, en el hospital binacional de macará ecuador [Tesis de pregrado]. [Loja-Ecuador] Universidad Nacional De Loja; 2017.

13. Cartes, R. Pautas de chequeo, parte IV: STARD y CARE. Revista Chilena de Cirugía. 2016 